\title{
COMPORTAMIENTO DE LA POBLACION DE Avena fatua TRATADA CON GRAMINICIDAS POSTEMERGENTES ${ }^{1}$
}

\author{
VICTOR F. JUAN ${ }^{2}$, J. H. IRIGOYEN ${ }^{3}$ y G. A. ORIOLI ${ }^{3}$
}

\begin{abstract}
En el sudoeste bonaerense de la República Argentina, Avena fatua es una maleza que ocupa una significativa proporción del banco de semillas presentes en el suelo, causando severas pérdidas de rendimiento en los cultivos de trigo, lo cuál la caracteriza como una maleza invasora. A pesar de que en los cultivos, se utilizan normalmente prácticas de control con graminicidas selectivos, se observa una alta incidencia de la población de Avena fatua a través de la determinación del número de panojas y semillas por metro cuadrado remanentes de las plantas que escapan al control y que alcanzan el estado reproductivo antes de la cosecha del cereal. El objetivo de este estudio fue evaluar el efecto de varios graminicidas postemergentes selectivos para el cultivo de trigo sobre el crecimiento de la población de Avena fatua por metro cuadrado en un diseño estadístico de bloques al azar con cuatro repeticiones. Los tratamientos fueron los siguientes: testigo sin control, tralkoxydim (150 y $200 \mathrm{~g} / \mathrm{ha})$, fenoxaprop-p-etil ( 69 y $83 \mathrm{~g} / \mathrm{ha}$ ), pirifenop-n-butil (50 y 55 $\mathrm{g} / \mathrm{ha}$ ) y diclofop metil (510 y $570 \mathrm{~g} / \mathrm{ha}$ ). En la primer cam-
\end{abstract}

RESUMEN paria, con una baja infestación de la maleza (153 plantas/m2), todos los herbicidas y dosis probadas ejercieron una drástica reducción de los parámetros evaluados, no detectándose diferencias significativas entre sí al evaluar el control por medio del número de panojas y semillas producidas por las plantas que escaparon al control. En cambio en la segunda campaña con 285 plantas $/ \mathrm{m}^{2}$ y habiéndose realizado la aplicación de los avenicidas en un estado fenológico de la maleza más avanzado, el número de semillas por metro cuadrado fue reducido a niveles que oscilaron entre un $60 \%$ y un $95 \%$ respecto del testigo sin control, detectándose diferencias entre los tratamientos. No obstante la reducción lograda, se comprobó en ciertos casos, un importante aporte de semillas que anualmente retornan al suelo, lo que podría significar sino un aumento, por lo menos un mantenimiento del número de propágalos en el banco.

Palabras claves: Control de Avena fatua, tralkoxy dim, diclofop-metil, fenoxaprop-p-etil, pirifenop-N-butil.

\section{ABSTRACT \\ Effect of post-emergence graminicides on the control of Avena fatua}

Wild oats is an important weed in wheat in the southwestern Pampas of Argentina representing a high percentage of the soil seed bank. Even though specific herbicides are used, a high number of panicles and seeds survive. The aim of this study was to evaluate the effect of post emergence selective graminicides upon the development of the wild oats seed bank in a wheat crop. The experiment was conducted in a randomized block with four replications. The treatments were: a) control (no herbicides), b) tralkoxydim (150 and $200 \mathrm{~g} / \mathrm{ha}$ ), c) fenoxaprop-p-etil (69 and $83 \mathrm{~g} / \mathrm{ha})$, d) pirifenop-n-butil (50 and $55 \mathrm{~g} / \mathrm{ha}$ ), and e) diclofop metil (510 and $570 \mathrm{~g} / \mathrm{ha}$ ). All herbicides were used at the recommended rates. In the first year, with a low infestation level (153 plants/m2), all herbicides had a good effect. No differences between treatments were observed. In the second year, with a high level (285 plants/m2), the production of wild oats seeds was reduced to between $60 \%$ and $95 \%$ compared with the control and there were differences between treatments. Despite the effectiveness of the treatments, the seeds produced were enough to keep the seed bank constant.

Additional index words: Avena fatua control, tralkoxydim, fenoxaprop-p-etil, diclofop-metil, pirifen op-Nbutil. 


\section{INTRODUCCION}

Avena fatua es una maleza anual, perteneciente a la familia de las gramíneas de alta habilidad competitiva. Posee diversas estrategias de supervivencia y perpetuación, lo que la caracteriza como una especie difícil de controlar. Se encuentra ampliamente difundida en los cultivos de trigo y otros cereales en varios paises del mundo causando en éstos severas pérdidas de rendimiento por competencia y disminuición del valor comercial del producto cosechado (Bowden \& Friesen, 1967; Bell \& Nalejawa, 1968; Chancellor et al., 1976; McNamara, 1976; García Baudín, 1982; Wilson \& Peters, 1982; López, 1983; O'Donovan \& Sharma, 1983).

En Argentina esta maleza es uno de los principales factores limitantes de la producción en una amplia región del sudoeste de la provincia de Buenos Aires y parte de La Pampa, ocupando una gran proporción de la subregión ecológica triguera $\mathrm{V}$ sur y extendiéndose hacia algunos partidos de la subregión IV, donde presenta grados de infestación variables (López, 1983).

A pesar de las prácticas de control utilizadas normalmente en el cultivo de trigo, frecuentemente con graminicidas de postemergencia, no se ha logrado reducir los niveles de infestación de ésta maleza.

Desde que Harper (1977) introduce la idea del suelo como un banco de semillas, numerosos estudios han demostrado la importancia del mismo en las estrategias de supervivencia y perpetuación de diversas especies. Tal es así que más recientemente Radosevich \& Holt (1984) destacan que el mayor componente de una población de malezas encontradas en un campo probablemente, tengan su origen en el depósito de semillas almacenadas previamente en el suelo, más que en las semillas recientemente diseminadas.

El objetivo de éste trabajo fué determinar el efecto de diversos graminicidas postemergentes utilizados en el cultivo de trigo sobre la población de Avena fatua a través de la determinación del número de panojas y de semillas por metro cuadrado remanentes de las plantas que escapan al control y que alcanzan el estado reproductivo antes de la cosecha del cereal.

\section{MATERIAL Y METODOS}

Los ensayos fueron realizados durante las campañas 1989 y 1990, en un predio ubicado en la localidad de Calderón, distante aproximadamente a $15 \mathrm{~km}$ de la Ciudad de Bahía Blanca, Provincia de Buenos Aires, República Argentina. Cada campaña correspondió a diferentes lotes provenientes de barbecho y cuyo manejo es la monocultura de trigo durante períodos de 3 a 4 años sucesivos y un año sin cultivo. Los lotes fueron sembrados con el cultivar Buck Poncho y cuando el cultivo alcanzó el estado de tres hojas, 13-20 según escala decimal de Zadoks et al. (1976), presentaron infestaciones de 153 y 285 plantas de Avena fatua por metro cuadrado para los períodos mencionados respectivamente.

Fueron realizados los siguientes tratamientos:

T0: Testigo sin control

T1: Tralkoxydim (Grasp C.E. 10\%) $150 \mathrm{~g}$ i.a./ha

T2: Tralkoxydim (Grasp C.E. 10\%) $200 \mathrm{~g}$ i.a./ha
Fl: Fenoxaprop-p-etil (Puma C.E. 6.9\%) 69 g i.a./ha

F2: Fenoxaprop-p-etil (Puma C.E. 6.9\%) 83 g i.a./ha

Pl: Pirifenop-n-butil (March C.E. 10\%) $50 \mathrm{~g}$ i.a./ha + tenso-

activo no jónico al 0,2\% "SANDOWET"

P2: Pirifenop-n-butil (March C.E. 10\%) 55 g i.a./ha + tensoactivo no iónico al 0,2\% "SANDOWET"

Dl: Diclofop metil (Iloxan C.E. 28.4\%) $510 \mathrm{~g}$ i.a./ha

D2: Diclofop metil (Iloxan C.E. 28.4\%) $570 \mathrm{~g}$ i.a./ha

Las aplicaciones fueron realizadas mediante un pulverizador manual tipo mochila, equipado con picos de abanico plano 80015 asperjando un volumen de solución equivalente a 150 1/ha. Las mismas fueron realizadas en dos estados fenologicos distintos de la maleza. En la campaña 1989 la totalidad de las plantas de Avena fatua se encontraban en estado de 2 a 3 hojas. En 1990 un 50\% de las plantas se presentaron entre 2 a 3 hojas y el resto en 3 a 4 hojas con 1 a 2 macollos. El estado fenológico del cultivo fue, en ambos casos, 14-21 según la escala decimal de Zadoks et aí.(1976). El control de latifoliadas fue realizado en todo el ensayo con bromoxinil una semana antes del tratamiento con graminicidas.

El diseño estadístico utilizado fué de bloques al azar con 9 tratamientos y 4 repeticiones; los datos fueron procesados estadísticamente mediante análisis de varianza y las medias comparadas entre sí por SNK test con p 0,05.

Para las evaluaciones fueron utilizados algunos de los criterios enunciados por Fernández-Quintanilla et al.(1986): conteo de plantas no afectadas por los tratamientos a los 30 días posteriores a la aplicación, panojas por metro cuadrado y producción de semillas por panoja, logradas a cosecha.

\section{RESULTADOS Y DISCUSION}

En la figura 1, pueden observarse los resultados de la eficacia de control de cada uno de los tratamientos involucrados en el ensayo expresado como porcentaje de plantas muertas deter-minadas en función de la diferencia entre la densidad inicial (antes del tratamiento) y la existente a los 30 días de la aplicación. En la campaña 1989, en la cuál la infestación re-sultó moderada y no existió una alta variabilidad de estados fenológicos de la maleza, todos los tratamientos proporcionaron controles superiores al $75 \%$, resultando fenoxaprop-p-etil en ambas dosis el único que demostró un control significativamente superior al resto. En la campaña 1990, en la cuál la infestación fue más severa y hubo diversidad de estados fenológicos en la población de Avena fatua, se pudo apreciar mayores diferencias entre los herbicidas. De cualquier manera, si bien la do-sis mas alta de fenoxaprop-petil presentó controles superiores a los demás tratamientos, la eficacia del control fué menor que en la campaña 1989.

Tomando como base la infestación inicial existente durante los dos períodos de ensayo, se observa que ante infestaciones moderadas de Avena fatua y aún con controles de alrededor de un $80 \%$, en los tratamientos Tl, P1 y D1 las plantas remanentes aportan al banco de semillas una cantidad de propágalos aproxi-madamente igual a la densidad inicial de plantas (Figura 2). Con altas infestaciones solo se obtuvo una merma importante de la producción de semillas que 


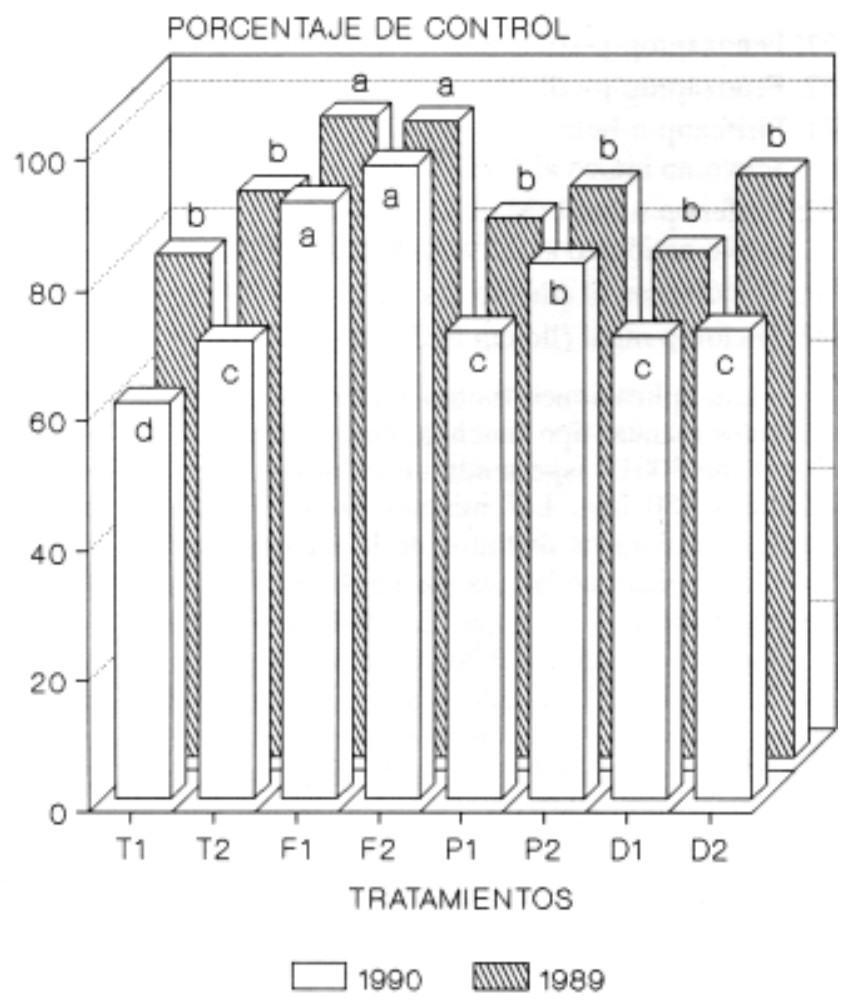

FIG. 1 - Eficacia de control de Avena fatua a los 30 días de la aplicación.

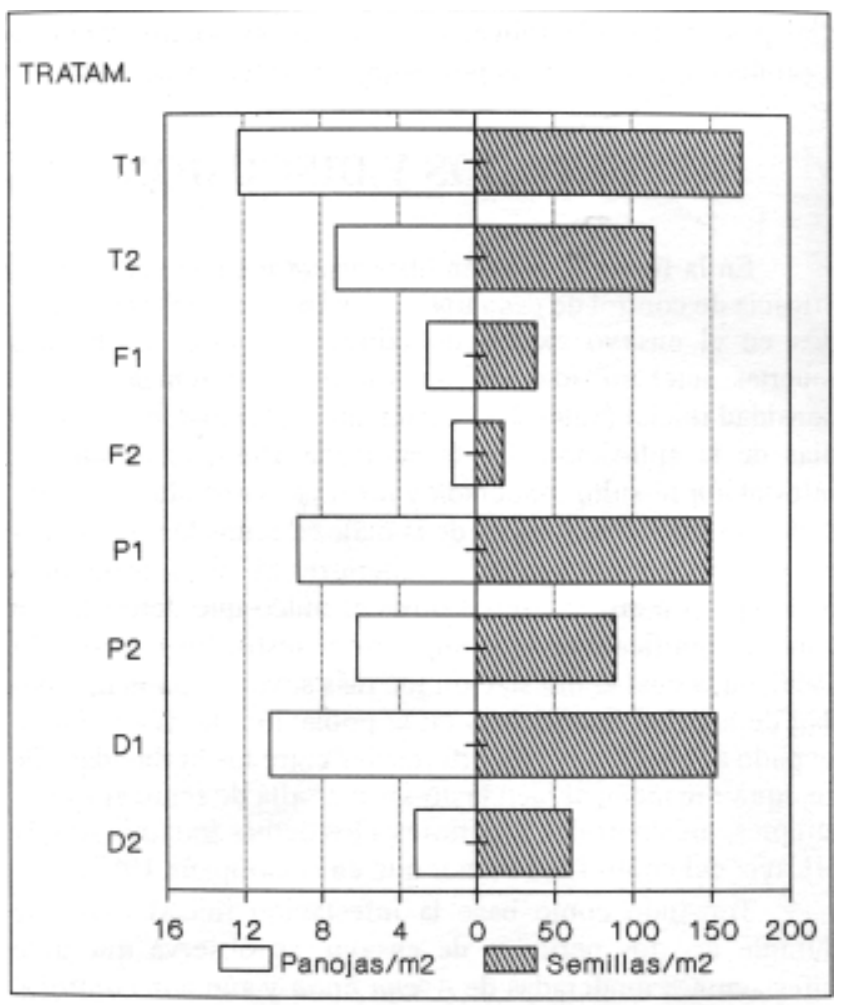

TESTIGO: 161 panoj./m2 y 2408 sem./m2

FIG. 2 - Número de panojas y semillas por metro cuadrado de la cosecha, obtenidos en la campańa 1989. vuelven al suelo respecto de la densidad inicial en los tratamientos Fl y F2 (Figura 3).

En efecto durante la campaña 1989 el testigo sin control produjo 15 semillas por panoja con una infestación inicial de 153 plantas por metro cuadrado. La aplicación de los graminicidas en el estado fenológico de 2 a 3 hojas, sin macollos, produjo una reducción tan importante del número de panojas que, aún cuando el número de semillas por panoja se mantuvo casi constante, los herbicidas de peor comportamiento solo permitieron un retorno de semillas similar al número de plantas iniciales.

En la campaña 1990, el testigo sin control produjo 22 semillas por panoja, con una infestación de 285 plantas por metro cuadrado y los graminicidas fueron aplicados en un estado feno-lógico más avanzado: $50 \%$ de plantas con macollos. En esas con-diciones se produjeron dos respuestas bien diferenciadas. Una fué la de los tratamientos Fl y F2 que redujeron tanto el número de panojas que aún manteniendo un alto número de semillas por panoja, produjeron un bajo número de propágulos. Otra fué la respuesta de los demás herbicidas que permitieron desarrollar un alto número de panojas que si bien tenían un número de semillas por panoja fuertemente reducido produjeron un importante retorno de propágulos. Aún con porcentajes de germinación mucho menores que los obtenidos en laboratorio (60 a 70\%) la incorporación de tan importante cantidad de propágulos al banco puede ser de significancia.

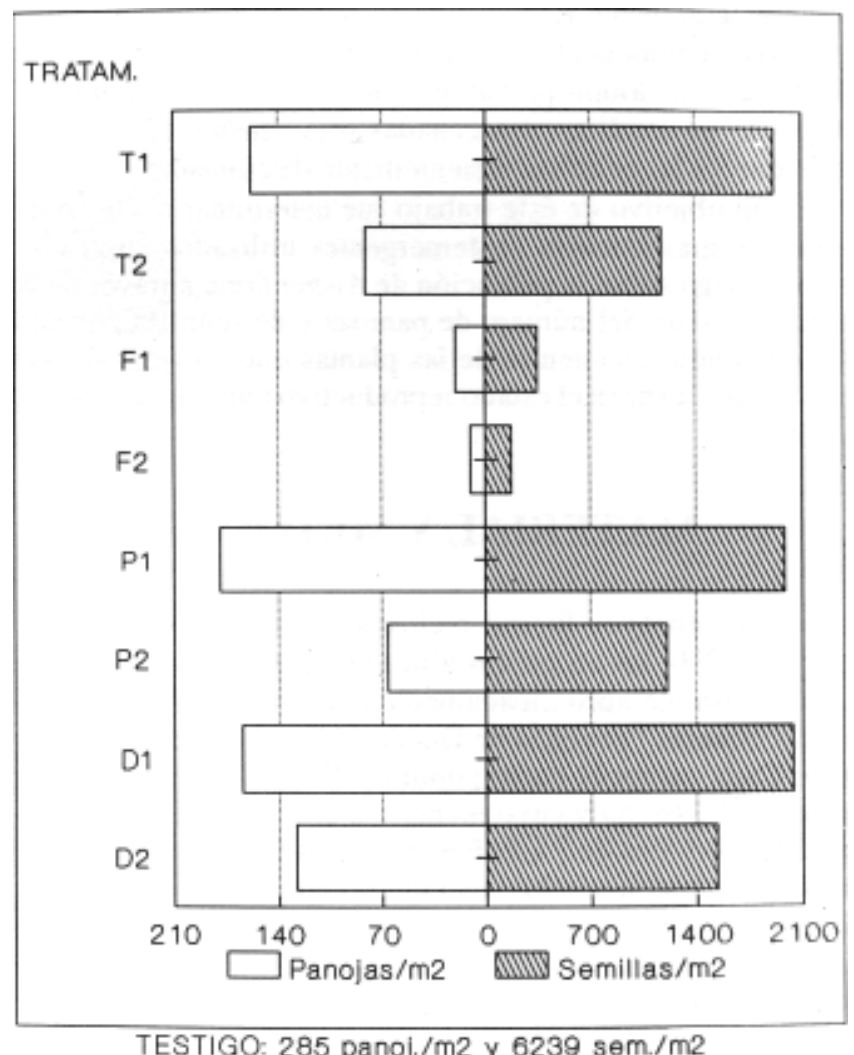

TESTIGO: 285 panoj./m2 y $6239 \mathrm{sem} . / \mathrm{m} 2$

FIG. 3 - Número de panojas y semillas por metro cuadrado de la cosecha, obtenidos en la campaña 1990. 
En condiciones de producción la aplicación anual sistemática de graminicidas raramente ocurre. Es más puede ocurrir que las aplicaciones al cultivo se realicen en momentos fenológicos y/o climatológicos no adecuados que no permitan obtener la mejor respuesta. Por lo tanto es difícil encontrarse con situaciones como las que destacan Fernández-Quintanilla et a!.(1987) al referirse a la influencia de ciertos tratamientos con herbicidas sobre la dinámica de la población de Avena spp. Ellos destacan que la aplicación anual de avenicidas provoca una reducción gradual del banco de semillas presentes en el suelo como así también del tamaño de sus poblaciones a largo plazo. Por el contrario, los datos aquí presentados comprueban que los altos porcentajes de control considerados eficientes desde el punto de vista productivo (Juan et al., 1991), pueden dejar una cantidad de plantas remanentes que producen macollos fértiles capaces de desarro-llar panojas con una cantidad importante de semillas que retoman al suelo. Este aumento o por lo menos mantenimiento del nú-mero de propágulos en el banco de semillas, sería la causa de que a pesar de los controles realizados normalmente en los cultivos de trigo, no se ha podido reducir aún la incidencia deAvena fatua en diversas zonas de nuestro país.

\section{LITERATURA CITADA}

BELL, A.R., NALEWAJA J.D. Competition of wild oat in wheat and barley. Weed Science, v. 16, p. 505-508, 1968.

BOWDEN, B.A.; FRIESEN, G. Competition of wild oat (Avena fatua L.) in wheat and flax. Weed Research, v. 7, p. 349-359, 1967

CHANCELLOR, R.J.; PETERS, N.C.P. Competition between wild oats and crops. In: JONES, D.P. (ed.) Wild oats in world agriculture. London: Agr. Res. Coun, 1976. p. 99-112.
FERNÁNDEZ-QUINTANILLA， C.; NAVARRETE， L.; TORNER, C. Criterios de evaluación en ensayos de herbicidas contra avena loca (Avena spp.) en cereales. Investigación Agraria, v. 1, n. 3, p. 379-392, 1986.

FERNÁNDEZ-QUINTANILLA， C.; NAVARRETE, L.; TORNER C.; ANDUJAR J.L. Influence of herbicide treatments on the population dynamics of Avena sterilis ssp. ludoviciana (Durieu) Nyman in winter wheat crops. Weed Research, v. 27, p. 375- 383, 1987.

GARCÍA BAUDÍN, J.M. Importancia de las avenas locas en España. Bol. Serv. Plagas, p. 35-42, 1982.

HARPER, J.L. The population biology of plants. London: Academic Press, 1977.

JUAN, V.F.; IRIGOYEN J.H.; ORIOLI, G.A. Eficacia de varios graminicidas postemergentes para el control de Avena fatua en trigo. In: REUNIÓN ARGENTINA SOBRE LA MALEZA Y SU CONTROL, 12, 1991. Resúmenes, 1991. p. 8.

LÓPEZ, R. Evaluación de distintos momentos de aplicación de herbicidas postemergentes para el control de Avena fatua (L.) en trigo. Malezas, p.73-89, 1983.

McNAMARA, D.W. Wild oat density and the duration of wild oat competition as it influences wheat growth and yield. Australian Journal Experimental Agriculture and Animal Husbandry, p. 402-406, 1976.

ODONOVAN, J.T.; SHARMA, M.P. Wild oats, competition and crop losses. In: Wild Oat Action Comm. Proc., Regina Saskatchewan. p. 27-37, 1983.

RADOSEVICH, S.R.; HOLT, J.S. Weed ecology: implications for vegetation management. New York: J. Wiley, 1984.

WILSON, B. J., PETERS N. C. B. Some studies of competition between Avena fatua L. and spring barley I. The influence of $A$. fatua on yield of barley. Weed Research, v. 22, p. 143-148, 1982. 